\title{
Esotropia do adulto durante o período gestacional: relato de caso
}

\author{
Adult esotropia during pregnancy:case report
}

\author{
Luciana Negrão Frota de Almeida ${ }^{1}$ \\ Miguel Gustavo Rosa da Rocha Canêdo ${ }^{2}$ \\ André Luis de Sousa Martins ${ }^{3}$ \\ Ricardo Gonçalves da Silva ${ }^{4}$ \\ Aclécio José Dias Pereira ${ }^{5}$
}

\section{RESUMO}

Os autores relatam o caso de uma paciente que apresentou quadro reincidente de esotropia aguda e diplopia durante o período gestacional. Não havia história de trauma ou qualquer alteração sistêmica, excetuando-se a gravidez. Tratamento oclusivo alternado foi instituído, e após o parto houve remissão espontânea dos sinais e sintomas.

Descritores: Estrabismo; Esotropia; Complicações na gravidez; Diplopia; Relatos de casos [Tipo de publicação]

\section{INTRODUÇÃO}

Entende-se por esotropia adquirida do adulto todo esodesvio ocorrido após os oito anos de idade, portanto após o período de plasticidade sensorial, que aparece em decorrência de diversos fatores: esotropias intermitentes que se tornam permanentes; microtropias descompensadas; alterações neurológicas; paresias ou paralisias musculares; após choque emocional (esotropia psicogênica) etc.

Dentre as várias formas de esotropias no adulto, destaca-se a esotropia aguda do adulto (EAA), uma forma rara de desvio que não apresenta etiologia definida, caracterizada por início tardio, presença de diplopia e comitância. Geralmente ocorre após um período de oclusão monocular para tratamento de um trauma ocular, por exemplo, o que permite o aparecimento da esotropia (ET) com diplopia. A maioria dos casos não melhora com tratamento clínico, necessitando intervenção cirúrgica ${ }^{(1-5)}$.

Neste artigo descreve-se um caso de esotropia do adulto de remissão espontânea durante o período gestacional sem indícios de alterações neurológicas ou sistêmicas.

\section{RELATO DO CASO}

Paciente do sexo feminino N.F.C., 29 anos, casada, procedente de Goiânia, procurou o serviço de urgência do Hospital da Fundação Banco de Olhos de Goiás relatando diplopia de início súbito e cefaléia frontal.

Referia quadro semelhante há quatro anos durante a primeira gravidez (a partir da $28^{\underline{a}}$ semana) ocasião em que foi submetida a exame neurológico, o qual se apresentava normal, e oftalmológico o qual revelava miopia, esotropia e diplopia. Fez tratamento oclusivo e ortóptico durante o período de gestação e, aproximadamente duas semanas após o parto, a miopia, o desvio e a diplopia haviam desaparecido.

$\mathrm{Na}$ avaliação geral apresentava somente atraso menstrual (20 dias). Negava trauma e doenças sistêmicas. 
Ao exame oftalmológico a acuidade visual sem correção era de 20/20 no olho direito (OD) e 20/20 no olho esquerdo (OE). A paciente referia diplopia a qualquer distância. O "Cover test" mostrou ET' de 15 dioptrias prismáticas $(\Delta)$ e HT' e/d de $5 \Delta$, com preferência pelo OD. Nas versões observou-se leve hipofunção do músculo reto lateral (RL) do OD e leve hiperfunção do músculo oblíquo superior (OS) em ambos os olhos. O ponto próximo de convergência era de cinco centímetros. O restante do exame oftalmológico apresentava-se sem alterações. Foi, então, solicitado teste ortóptico (Quadro 1A e 1B), exame neurológico e prescrito oclusão 1:1.

A paciente realizou oclusão somente do OD durante o dia até aproximadamente a $13^{\underline{a}}$ semana de gestação quando apresentou melhora do quadro. Após aproximadamente três meses sem oclusão e sem apresentar diplopia ou desvio, a paciente retorna ao nosso serviço com reaparecimento do quadro clínico, que permaneceu inalterado até o final da gravidez.

No $10^{\circ}$ dia de puerpério o exame estrabológico não apresentava alterações e o teste ortóptico revelava ortotropia em todas as posições do olhar.

\section{DISCUSSÃO}

Apresenta-se um quadro de ET do adulto de início súbito, em uma paciente emétrope, sem sinais de neuropatia, que possui como fator desencadeante do desvio o estado gestacional. Torna-se difícil a classificação deste desvio, uma vez que a esotropia aguda do adulto é uma forma rara e bem definida de estrabismo e a ET psicossomática, que também pode ocorrer de forma aguda, apresenta alguns sinais não compatíveis com o quadro.

Dois tipos distintos de esotropia aguda do adulto têm sido descritos: esotropia tipo Franceschetti e esotropia tipo Bielschowisky.

\section{Quadro 1A}

4A de Jampolsky: supressao do OE

Filtro vermelho: diplopia homônima

Para longe: fusão com $20 \Delta$ base externa no OD

Para perto: fusão com $16 \Delta$ base externa no OD

Versões: $-1,0 \mathrm{RL}$ e $+0,5$ oblíquo superior $\mathrm{OD}$

$-0,5 \mathrm{RL}$ e $+0,5$ oblíquo superior $\mathrm{OE}$

\begin{tabular}{|c|c|c|}
\hline \multicolumn{3}{|c|}{ Quadro 1B } \\
\hline \multicolumn{3}{|c|}{ Posições diagnósticas para longe sem correção } \\
\hline ET $20 \Delta$ & ET $18 \Delta$ & ET $10 \Delta$ \\
\hline ET $25 \Delta$ & ET $20 \Delta$ & ET $8 \Delta / \mathrm{HT}$ e/d $3 \Delta$ \\
\hline ET $20 \Delta$ & ET $16 \Delta$ & ET $6 \Delta / \mathrm{HT}$ e/d $3 \Delta$ \\
\hline \multicolumn{3}{|c|}{ Posições diagnósticas para perto sem correção } \\
\hline ET' $20 \Delta$ & $E T^{\prime} 20 \Delta$ & ET' $8 \Delta$ \\
\hline ET' $20 \Delta / \mathrm{HT}$ e/d $2 \Delta$ & ET' $18 \Delta / \mathrm{HT}$ e/d $2 \Delta$ & $\mathrm{ET}^{\prime} 6 \Delta / \mathrm{HT}$ e/d $4 \Delta$ \\
\hline ET' $18 \Delta$ & ET' $16 \Delta$ & $\mathrm{ET}^{\prime} 5 \Delta / \mathrm{HT}$ e/d $3 \Delta$ \\
\hline
\end{tabular}

A esotropia aguda do adulto do tipo Bielschowisky é observada em pacientes míopes, usualmente neuropatas. Observa-se esotropia e diplopia para longe e ortotropia para perto. A patogenia é desconhecida. Tem-se relacionado esse tipo de desvio com transtornos na divergência tônica, doenças do sistema nervoso central ou paresia bilateral do abducente. A conduta expectante ou o tratamento clínico com prisma e expectante podem ser realizados, especialmente em pacientes com alterações neurológicas, devido à possibilidade de melhora da esotropia nesses casos. No entanto a grande maioria deles necessita de tratamento cirúrgico ${ }^{(1-3,6-7)}$.

A esotropia tipo Franceschetti ocorre em indivíduos jovens, emétropes ou ligeiramente hipermétropes, sem sinal de neuropatia. A esotropia surge geralmente após um curto período de oclusão monocular para tratamento de um trauma ocular, por exemplo, o que sugere uma esoforia descompensada. A diplopia é percebida a qualquer distância e o desvio se mostra de pequeno a médio ângulo com perfeita comitância. Como o tratamento clínico com prismas ou exercícios ortópticos são insuficientes, o tratamento cirúrgico se faz necessário, na maioria das vezes, com bom prognóstico ${ }^{(2)}$.

No caso em questão, a paciente apresenta sinais sugestivos de uma EAA do tipo Franceschetti, no entanto dois fatores importantíssimos são discordantes da literatura, primeiro porque não houve trauma ocular ou uso de oclusivo que proporcionasse o aparecimento de uma esoforia descompensada; segundo que houve melhora espontânea da ET e da diplopia, fato jamais descrito neste tipo de esotropia.

É importante salientar que muito embora prismas de Fresnel possam ser usados para alívio da diplopia ${ }^{(1-2)}$, a oclusão alternada foi escolhida como opção terapêutica em vista da dificuldade de aquisição desse tipo de prisma.

Questiona-se, em seguida, a possibilidade de tratar-se de uma esotropia psicossomática. Este tipo de desvio é mais freqüente em crianças, entre sete e oito anos de idade, que sofreram algum tipo de choque emocional importante. O estrabismo nesses casos é intermitente e incomitante ${ }^{(4)}$ e geralmente apresenta um terreno receptivo para o aparecimento da ET, como uma esoforia subjacente ou uma hipermetropia que até então estava compensada ${ }^{(1,4,8)}$.

Entretanto, sabe-se hoje que as alterações psicossomáticas se apresentam das mais diversas formas, e apesar de não existir casos semelhantes na literatura, não se descartou a hipótese de tratar-se de um caso de estrabismo psicogênico, pois a paciente relatou insatisfação do marido com relação à gravidez, o que pode ter ocasionado uma instabilidade emocional.

É importante ressaltar que a alteração hormonal inerente ao quadro gestacional pode ter de, alguma forma, contribuído para o surgimento da ET, entretanto sua fisiopatogenia não foi esclarecida.

A classificação exata de que tipo de ET a paciente apresentava não foi possível, em vista de não existirem casos semelhantes na literatura e do desvio não apresentar alguns requisitos impor- 
tantes para classificá-lo em um tipo de ET já descrito. Concluiu-se que se tratava de uma esotropia do adulto cujo principal fator desencadeante encontrado foi o estado gestacional.

\section{ABSTRACT}

The authors report a case of a female patient who presented, for the second time, diplopia and acute esotropia during pregnancy. There was no trauma involved neither was systemic disease. The patient was treated with conventional occlusion, and, soon after delivery, the squint was resolved.

Keywords: Strabismus; Esotropia; Pregnancy complications; Diplopia; Case reports [Publication type]

\section{REFERÊNCIAS}

1. Duke-Elder S. Concomitant strabismus: Heterotropia. In: Duke-Elder S. editor. System of ophthalmology. London: Henry Kimpton; 1973. v.4. p.611-2.

2. Souza-Dias C, Díaz JP. Esotropias. In: Prieto-Díaz J, Souza-Dias C. Estrabismo. 4a ed. São Paulo: Livraria Santos Editora; 2002. p.149-99.

3. Sá LCF. Estrabismo nos anos 90. Arq Bras Oftalmol. 1998;61(6):720-4.

4. Hugonnier R, Hugonnier S. Estrabismos concomitantes convergentes. In: Hugonnier R, Hugonnier S, editors. Estrabismos - heteroforias paralysis oculomotrices. Barcelona: Masson; 1973. p.226-43.

5. Miller NR, Lee AG. Adult-onset acquired oculomotor nerve paresis with cyclic spasms: relationship to ocular neuromyotonia. Am J Ophthalmol. 2004;137(1):70-6.

6. Webb H, Lee J. Acquired distance esotropia associated with myopia. Strabismus. 2004;12(3):149-55.

7. Spierer A. Acute concomitant esotropia of adulthood. Ophthalmology. 2003; 110(5):1053-6.

8. Prata Júnior JA. Estrabismo psicossomático. Rev Bras Oftalmol. 1989;48(6): 381-3.

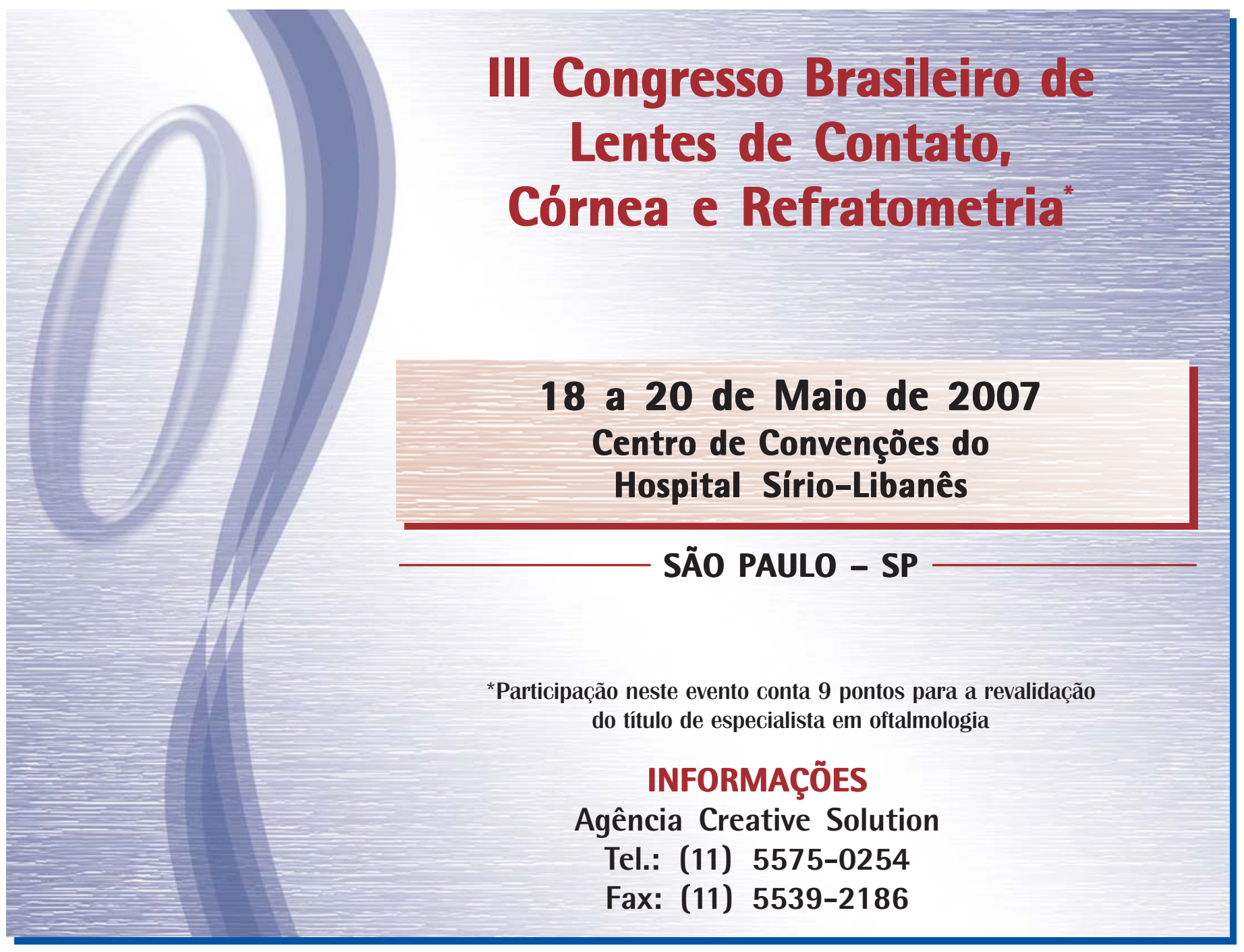

